\title{
A Comprehensive Performance Analysis of MIMO-OFDM Technology Using Different MIMO Configurations and M-QAM Modulation Schemes for LTE Cellular Network
}

\author{
Jobaida Akhtar ${ }^{1}$, Mohammad Istiaque Reja ${ }^{1}$, Md. Al Amin ${ }^{1} \&$ Md. Sahidur Rahman ${ }^{1}$ \\ ${ }^{1}$ Department of Electrical and Electronic Engineering, Chittagong University of Engineering and Technology, \\ Bangladesh \\ Correspondence: Jobaida Akhtar, Department of Electrical and Electronic Engineering, Chittagong University of \\ Engineering and Technology, Chittagong-4349, Bangladesh. Tel: 880-174-705-1590. E-mail: \\ lilyjobaida@cuet.ac.bd
}

Received: February 15, 2018

Accepted: February 28, 2018 Online Published: March 30, 2018

doi:10.5539/mas.v12n4p118

URL: https://doi.org/10.5539/mas.v12n4p118

\begin{abstract}
MIMO-OFDM (Multiple Input Multiple Output-Orthogonal Frequency Division Multiplexing) uses multiple antennas at transmitter and receiver side to facilitate high throughput performance without needing additional bandwidth or transmission power. But frequency synchronization, Inter Carrier Interference (ICI) minimization and antenna diversity are the main challenges for MIMO-OFDM wireless technology. In this paper, a MIMO-OFDM hybrid model is designed with proper frequency synchronization and antenna diversity that minimizes ICI with improved signal strength. Using the model a comprehensive analysis in terms of Bit Error Rate (BER) performance with respect to Signal to Noise Ratio (SNR) and Bit Rate for different M-ary QAM modulation schemes and different MIMO configurations are presented over Rayleigh fading and AWGN channel. After an extensive analysis, it is found that the propose OFDM scheme shows better Bit Error rate (BER) performance for 64-QAM than any other M-ary QAM modulation schemes and the optimum MIMO configuration for this good quality performance is found to be $3 \times 2$ configuration.
\end{abstract}

Keywords: MIMO, SNR, OFDM, M-ary QAM, ICI, BER, frequency synchronization

\section{Introduction}

The demand for a high speed wireless communication, which is spectrally efficient as well as reliable, is getting higher and higher by the explosive growth of wireless communication over the recent years. Facilitating this good quality service is difficult due to some challenges such as channel time variation and limited available bandwidth (Gupta, Gupta \& Saini, 2011; Gesbert, Shafi, Shiu, Smith \& Naguib, 2003; Jiang \& Hanzo, 2007; Van Zelst \& Schenk, 2004). In order to increase the capacity many antennas are used both at transmitter and receiver side for sending independent information. This system is called Multiple Input Multiple Output (MIMO) technology where in order to increase the gain diversity space time coding is exploited (Sahrab \& Marghescu, 2014). MIMO technology in combination with orthogonal frequency division multiplexing (OFDM), which is a multicarrier digital modulation scheme having high spectrum efficiency and high data rate along with the immunity to frequency selective fading and Inter Symbol Interference (ISI) unlike conventional single carrier modulation scheme, have received considerable attention of the researchers as a promising technique for next generation wireless communication (Van De Beek, Edfors, Sandell, Wilson \& Borjesson, 1995; Bhatnagar, Singh \& Tiwari, 2011; Yu \& Ottersten, 2002).

Sondhi et al evaluated the performance of MIMO-OFDM systems for QPSK modulation with the use of cyclic prefix, zero padding and equalization and found the BER of $10^{-6}$ for $10 \mathrm{~dB}$ Signal to Noise Ratio (SNR) (Sondhi \& Reena, 2016). Wu et al used a hybrid pilot assisted channel estimation algorithm in MIMO-OFDM for low SNR and found BER of $10^{-5}$ for $7 \mathrm{~dB}$ SNR (Wu, Kuang, Ni, Lu, Huang \& Guo 2014). Dai et al used the time-frequency training OFDM (TFT-OFDM) transmission scheme which provides BER of approximately $10^{-5}$ for $9 \mathrm{~dB}$ SNR in large scale MIMO systems (Dai, Wang \& Yang, 2013).

In this paper, we have proposed a MIMO-OFDM model over Rayleigh fading and Additive White Gaussian Noise (AWGN) channel with limited bandwidth spectrum. Orthogonal Space Time Block Code (OSTBC) has 
been used in MIMO Channel. Minimization of Inter Carrier Interference (ICI) and frequency synchronization are properly maintained by proper insertion of Cyclic Prefix, Pilot and Zero padding with the data streams. Subsequently using the developed MIMO-OFDM model a comprehensive comparative analysis is carried out among different M-ary QAM modulation schemes and different MIMO configurations in order to find out the combination of best modulation scheme and best MIMO configuration providing lowest Bit error rate (BER). This analysis will be extremely useful for the researchers to have a complete overview of the performance of MIMO-OFDM technology that uses M-ary QAM modulation schemes over Rayleigh fading and Additive White Gaussian Noise (AWGN) channel.

\section{Brief Theory}

\subsection{Multiple Input Multiple Output (MIMO) System}

Figure 1(a) shows a MIMO system which uses multiple antennas at the transmitter and receiver unlike conventional Single Input Single Output (SISO) system. Signal quality and system capacity is improved in MIMO by means of spatial diversity and spatial multiplexing.

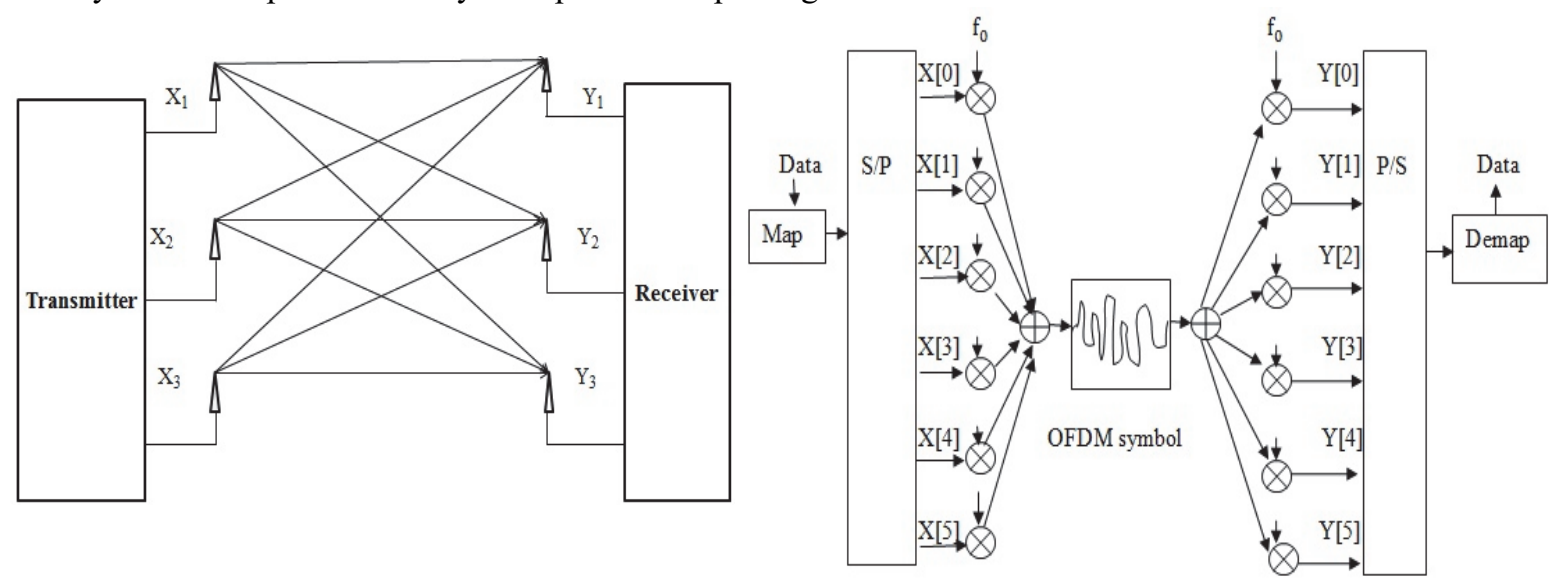

Figure 1. (a) MIMO Channel (b) Orthogonal Frequency Division Multiplexing

\subsection{Orthogonal Frequency Division Multiplexing (OFDM)}

OFDM is a technology where both the modulation and multiplexing are combined. To put it simply, many independent signals, which are sub-set of a single signal, are multiplexed in this technique. Instead of transmitting a single carrier having high data rate, OFDM transmits many orthogonal closely spaced subcarriers in parallel having low symbol rate (Medvedev, Bjerke, Walton, Ketchum, Wallace \& Howard, 2006). These subcarriers are modulated with a digital modulation scheme. Figure 1(b) illustrates the OFDM scheme. In receiver the reverse process are used to recover the data.

\section{kth OFDM symbol $\quad(k+1)$ th OFDM symbol}

Figure 2. OFDM symbols without guard interval

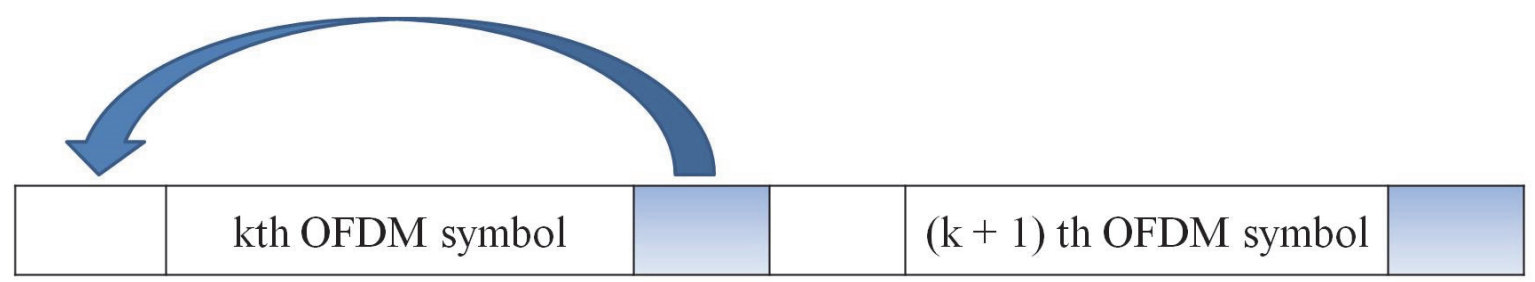

Figure 3. OFDM symbols with guard interval using $\mathrm{CP}$ technique 
Two consecutive OFDM symbols are shown in figure 2. The symbols do not have any guard interval. If the number of subcarriers is $\mathrm{n}$, the duration of one subcarrier is $\mathrm{t}_{\mathrm{s}}$ and $\Delta \mathrm{f}$ is the subcarrier spacing, then the effective OFDM symbol duration $t_{\text {sub }}$ can be written as (Dhore \& Hate, 2015):

$$
t_{\text {sub }}=n t_{s}=\frac{n}{\Delta f}
$$

The multipath fading channel effect on OFDM symbol can be reduced hugely if the symbol duration is increased by $\mathrm{n}$ times. But the orthogonality among the subcarriers may still break (Tian, Jiang, Song, Luo \& Xu, 2006). If a guard interval is introduced whose duration is longer than or equal to the maximum delay of the multipath channel then the orthogonality among the subcarriers can be retained (Dhore \& Hate, 2015). There are mainly two ways of introducing guard interval: Zero Padding (ZP) and Cyclic Prefix (CP) or Cyclic Suffix (CS). Filling up the guard intervals with the zeroes is known as zero padding, whereas if the last samples are copied to the starting of the OFDM symbol or first samples are copied to the ending of the OFDM symbol it is known as cyclic prefix or cyclic suffix respectively. Figure 3 shows how the guard interval is inserted into the two consecutive OFDM symbols by use of CP technique. If the duration of guard interval is tg, then the duration of the OFDM symbol will be (Dhore \& Hate, 2015):

$$
t_{\text {sym }}=t_{\text {sub }}+t_{g}
$$

\subsection{MIMO-OFDM}

Figure 4 shows the typical block diagram of an MIMO-OFDM transmitter and receiver. This figure shows a $2 \times 2$ MIMO configuration i.e. two transmitter antenna and two receiver antenna.

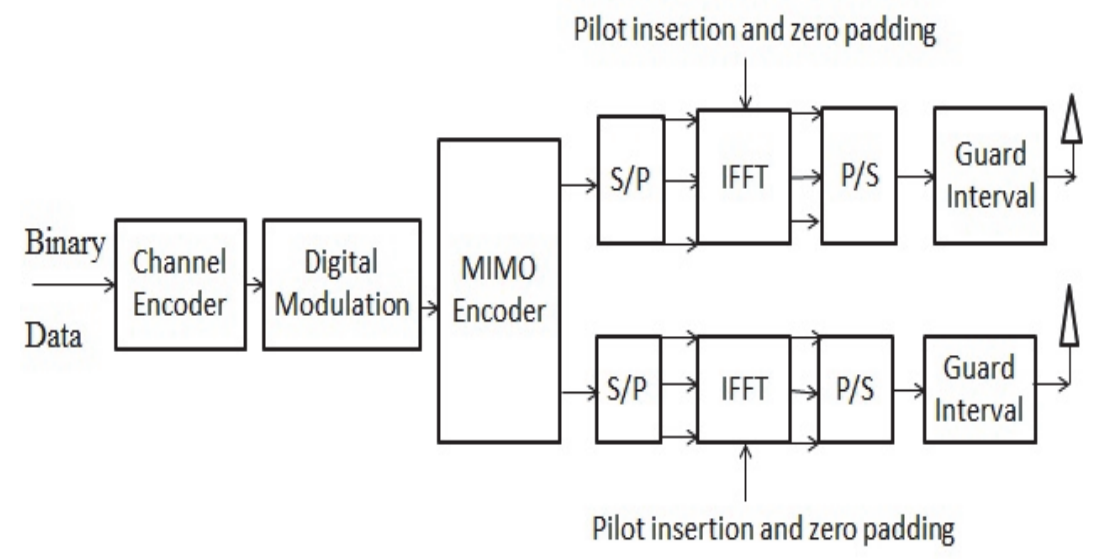

MIMO OFDM Transmitter

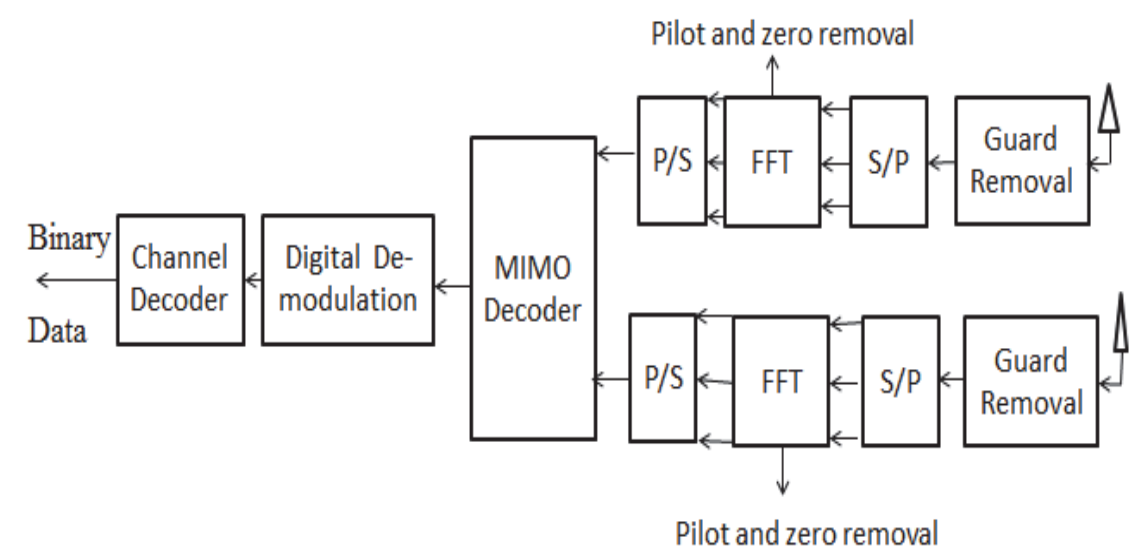

MIMO OFDM Receiver

Figure 4. Block Diagram of a typical MIMO-OFDM model (Maharaja, Mishra \& Bansode, 2016) 


\section{System Model}

A MIMO-OFDM model is implemented using the Simulink ${ }^{\circledR}$ as shown in figure 5. The model can be divided into four sections (OFDM transmitter, MIMO wireless channel, OFDM receiver, BER performance test). Data Source, IQ Mapper, OFDM Modulator are the blocks of OFDM transmitter. In MIMO wireless channel, Orthogonal Space Time Block Code (OSTBC) Encoder and Decoder, Rayleigh fading and AWGN channel are used. Data Sink, IQ Demapper and OFDM Demodulator make the OFDM receiver. In BER performance test section, total number of received bits, number of received error bits and packet loss are calculated and displayed. Several scopes like vector scope, spectrum scope are used in this proposed model to show the strength of signal.

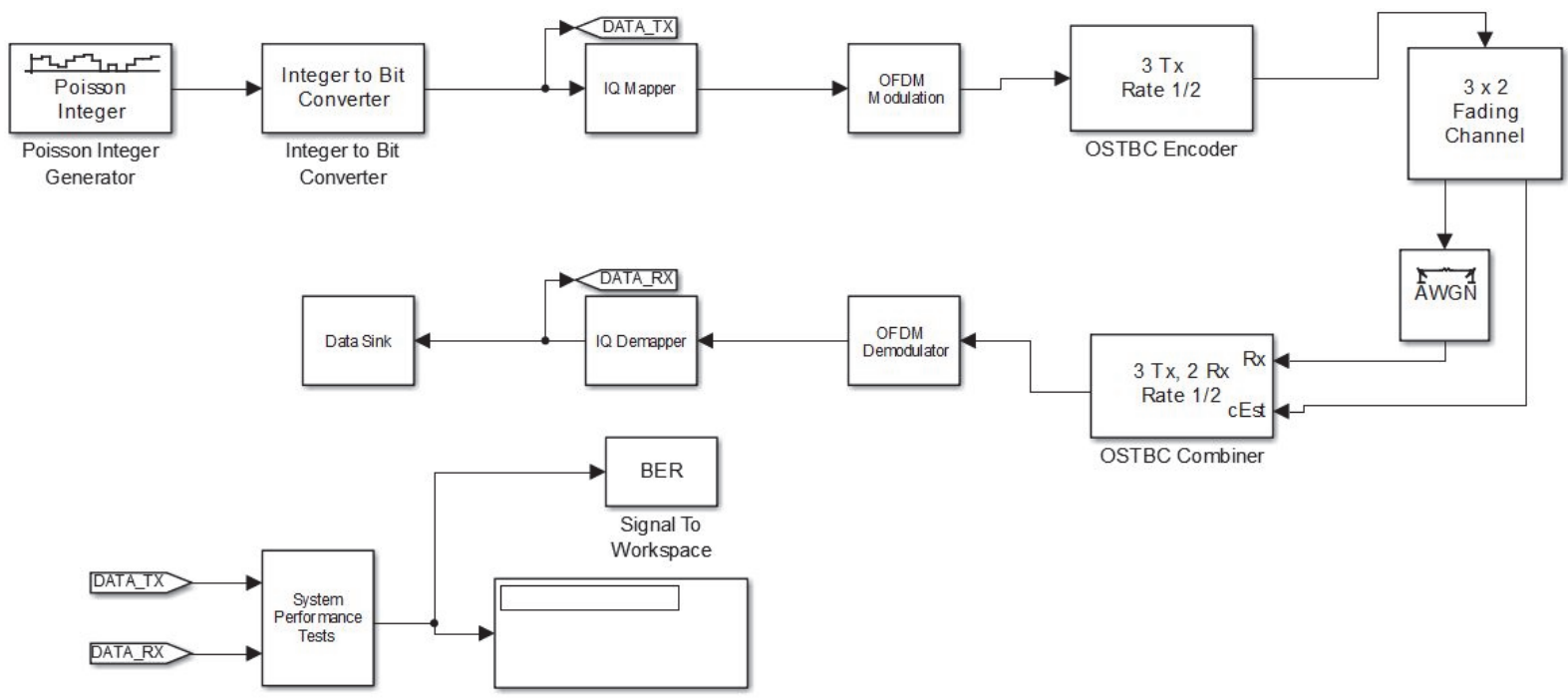

Figure 5. Implementation of the MIMO-OFDM model using Simulink ${ }^{\circledR}$

Fast Fourier Transform (FFT) calculation, Pilot and Zero Padding insertion are most important factors in order to maintain the frequency synchronization, removal of Inter Carrier Interference (ICI) in OFDM modulator Block. For $\mathbf{2 5 6}$ point FFT operation, the data streams are transmitted in $\mathbf{1 0}$ parallel paths. On the other hand, $\mathbf{1 1}$ parallel paths are used to insert pilot and zero padding that is shown in figure 6 . The total number of data streams zero padding are respectively 192 and $\mathbf{5 6}$. Table 1 summarizes all these information.

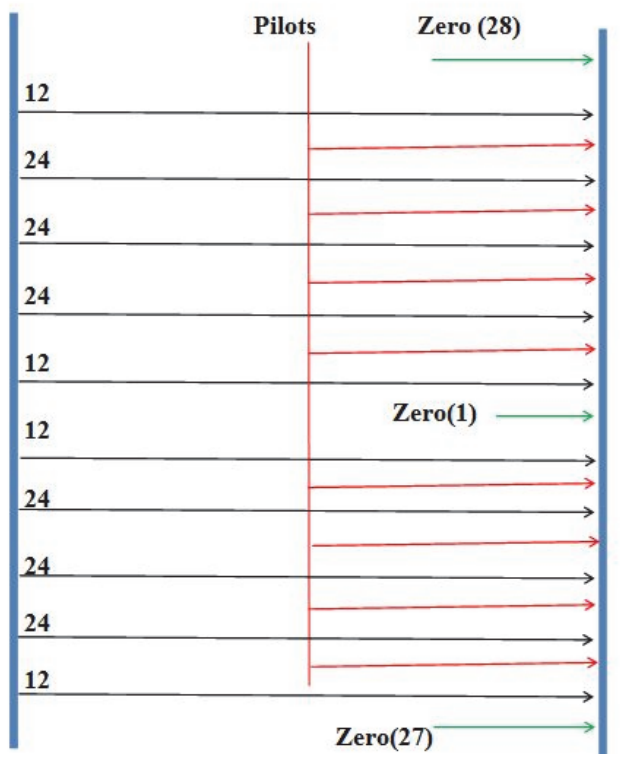

Figure 6. Insertion of data stream, zero padding and pilots for 256 point FFT 
Table 1. Data stream, zero padding and pilots calculation for 256 point FFT

\begin{tabular}{lc}
\hline \multicolumn{1}{c}{ Parameter } & Value \\
\hline Total number of parallel Paths for Data Stream & 10 \\
Total number of parallel Paths for Pilot & 08 \\
Total number of parallel Paths for Zero Padding & 03 \\
Number of Data Stream & $4 \times 12+6 \times 24=192$ \\
Number of Zero Padding & $28+1+27=56$ \\
\hline
\end{tabular}

Various parameters are used in every block to design MIMO-OFDM model. Different digital modulation schemes are used in IQ Mapper block. To obtain different MIMO configurations, the number of transmitter and receiver antennas are changed accordingly in OSTBC Encoder Block. The chosen design parameters to design MIMO-OFDM model are listed in Table 2.

Table 2. Simulation Parameters

\begin{tabular}{lc}
\hline \multicolumn{1}{c}{ Model Parameters } & Value \\
\hline Channel Bandwidth & $3 \mathrm{MHz}$ \\
Sampling frequency & $3.84 \mathrm{MHz}$ \\
FFT size & 256 \\
Samples Per Frame & 192 \\
Zero padding & 56 \\
Pilot & 8 \\
Cyclic Prefix & 64 \\
Symbol Period & $.26 \mu \mathrm{s}$ \\
Signal Power & $10 \mathrm{mw}$ \\
Code Rate & $1 / 2$ \\
Channel Type & Rayleigh, AWGN \\
Channel spacing & $15 \mathrm{kHz}$ \\
\hline
\end{tabular}

\section{Simulation and Result Analysis}

In this section the performance of the proposed OFDM-MIMO model is investigated in mobile multipath channels. For performance analysis, The BER vs. SNR (keeping the Bit Rate constant) and BER vs. Bit Rate (keeping the SNR constant) curves are plotted for different $\mathrm{m} \times \mathrm{n}$ MIMO configurations with different $\mathrm{M}$-ary QAM modulation schemes in order to find out the best modulation scheme and best MIMO configuration giving lowest BER.

Figure 7 shows the BER with respect to Signal to Noise Ratio (SNR) at a constant bit rate of $100 \mathrm{Mbps}$ for $2 \times 2$ MIMO configuration for different M-ary QAM schemes. From the figure, it can be seen that 64-QAM modulation scheme shows the best performance (minimum BER) and 8QAM modulation scheme shows the worst performance (maximum BER). For instance, for 6dB SNR 64-QAM provides BER of $2 \times 10^{-4}$ whereas 8-QAM provides BER of $10^{-2}$.

The BER with respect to SNR for $3 \times 2$ MIMO configuration for different M-ary QAM schemes are shown in figure 8. Here also the simulation is done at a constant bit rate of $100 \mathrm{Mbps}$. From the figure it can be seen that, 64-QAM shows better performance than other M-ary QAM in this MIMO configuration as in the case of $2 \times 2$ MIMO configuration. But, this time 32-QAM shows the poorest performance. For 6 dB SNR, 64-QAM provides BER of $10^{-7}$ whereas 32-QAM provides BER of $10^{-5}$. If we compare figure 7 and figure 8 , it is found that the proposed OFDM scheme performs better in $3 \times 2$ MIMO configuration than in $2 \times 2$ MIMO for all M-ary QAM modulation. 


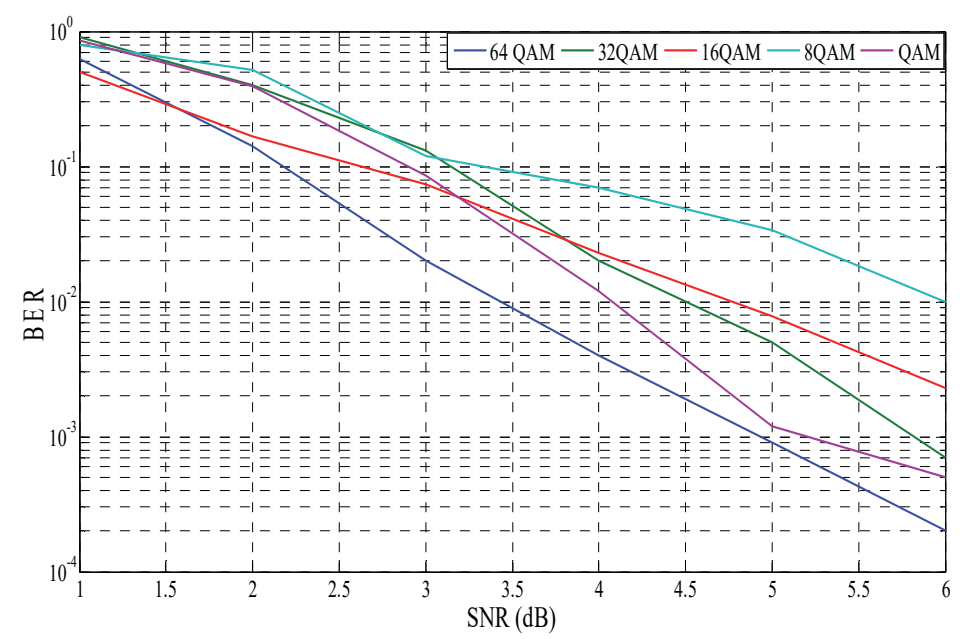

Figure 7. BER vs. SNR curves of $2 \times 2$ MIMO-OFDM for different M-ary QAM modulation

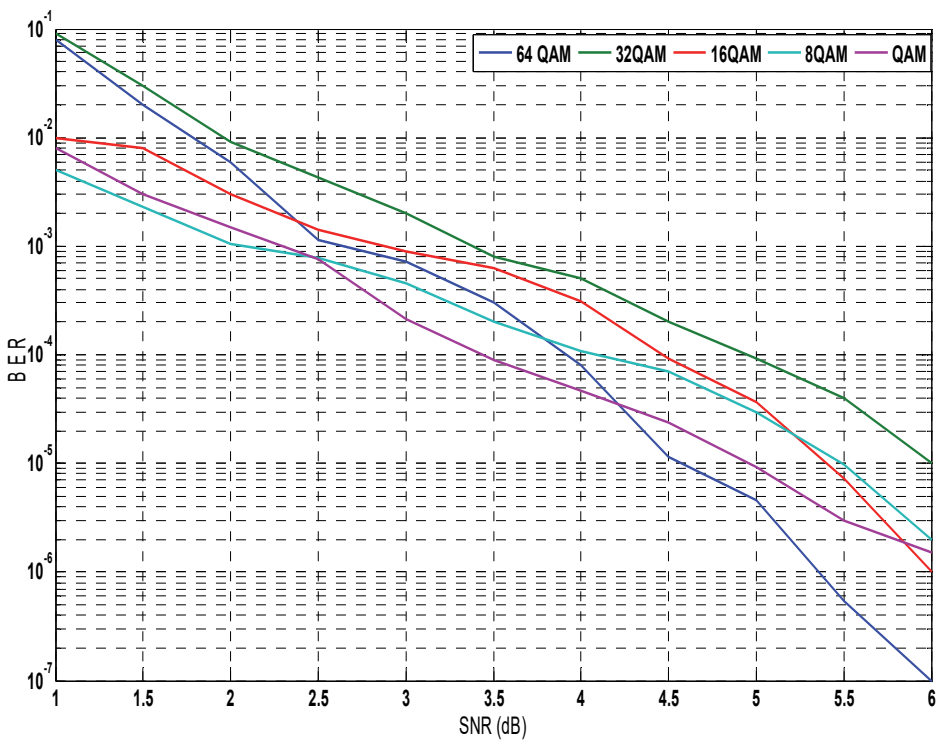

Figure 8 . BER vs. SNR curves of $3 \times 2$ MIMO-OFDM for different M-ary QAM modulation

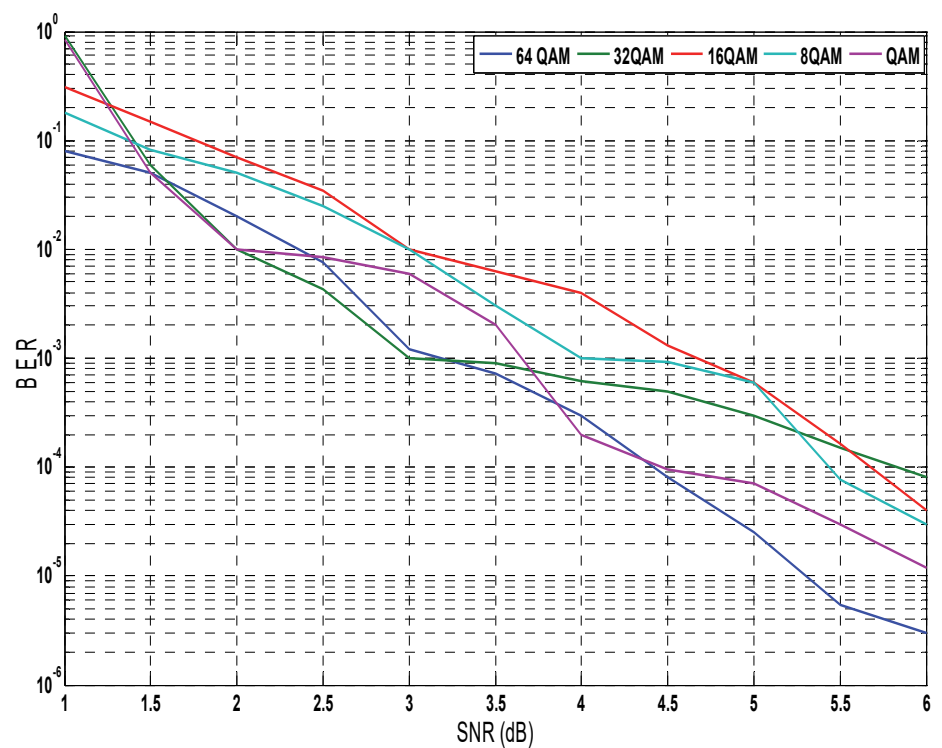

Figure 9. BER vs. SNR curves of $4 \times 3$ MIMO-OFDM for different M-ary QAM modulation 
Figure 9 shows the BER Vs SNR for 4×3 MIMO configuration. Here 8-QAM, 16-QAM and 32-QAM show poor BER in comparison to QAM and 64-QAM, which is in the range of $3 \times 10^{-5}$ to $8 \times 10^{-5}$ for $6 \mathrm{~dB}$ SNR. As in the case of previous two MIMO configurations, 64 QAM shows the minimum BER which is $3 \times 10^{-6}$. From figure 7 , figure 8 and figure 9 , it can be seen that the performance of proposed OFDM scheme in $4 \times 3$ MIMO configuration is better than $2 \times 2$ configuration and poorer than $3 \times 2$ configuration.

Figure 10 shows the BER with respect to Bit Rate at a constant SNR of $7 \mathrm{~dB}$ for $2 \times 2$ MIMO configuration for different M-ary QAM schemes. The BER is expectedly increasing with increasing bit rate. Here 64-QAM shows the best performance. For instance, for a Bit Rate of $80 \mathrm{Mbps}$ the BER of QAM and 8-QAM is in the range of $7 \times 10^{-4}$ to $9 \times 10^{-4}$, the BER of both $16-\mathrm{QAM}$ and 32 -QAM is $10^{-4}$, while $64-\mathrm{QAM}$ provides BER of $2 \times 10^{-5}$.

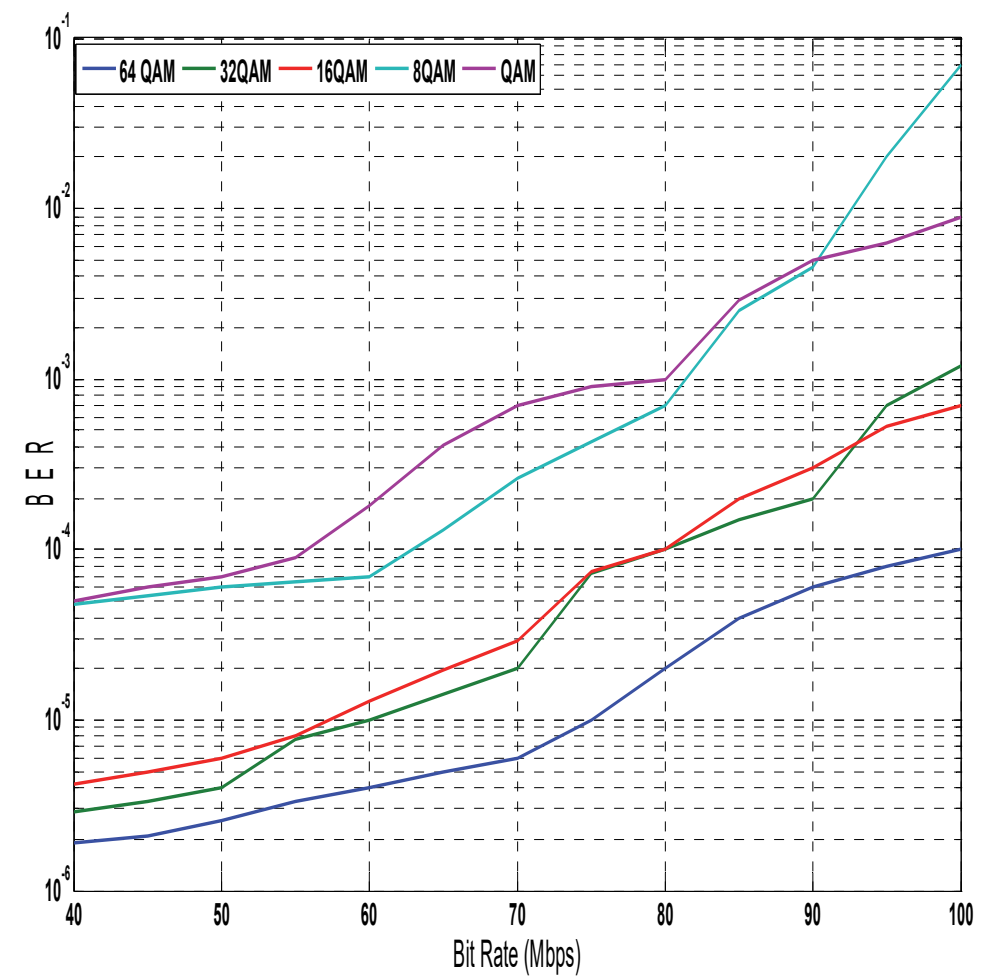

Figure 10. BER vs. Bit Rate curves of $2 \times 2$ MIMO-OFDM for different M-ary QAM modulation

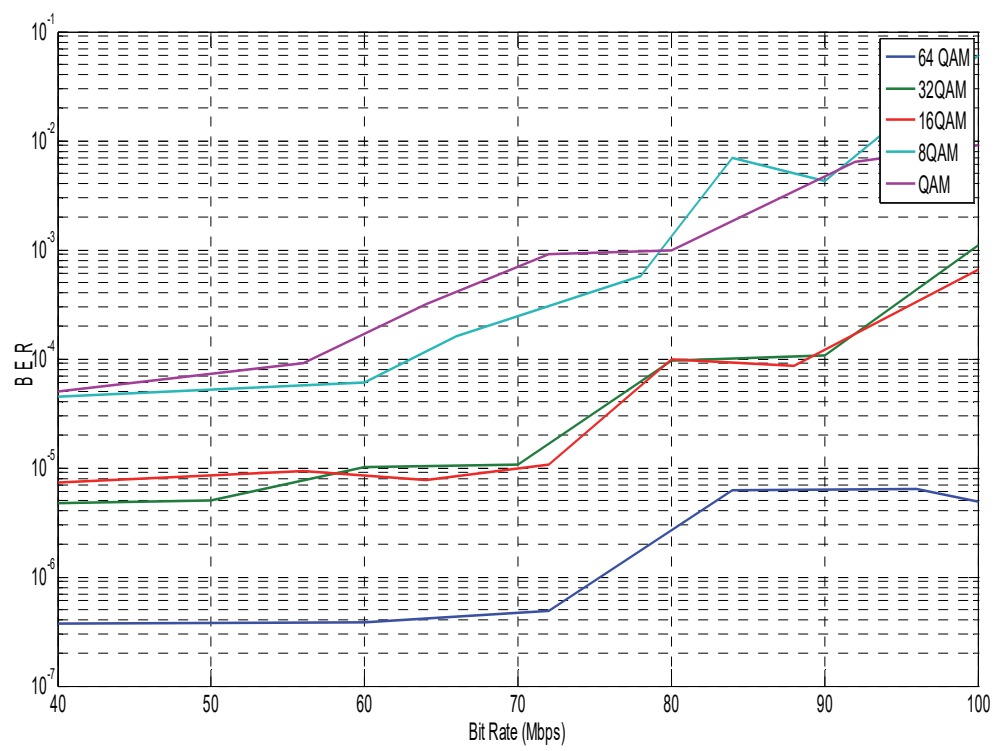

Figure 11. BER vs. Bit Rate curves of $3 \times 2$ MIMO-OFDM for different M-ary QAM modulation 
In $3 \times 2$ MIMO configuration, $100 \mathrm{Mbps}$ bit rate is achieved at a BER of $5 \times 10^{-6}$ using 64-QAM as shown in figure 11. At this bit rate 16-QAM and 32-QAM provides BER of $4 \times 10^{-4}$ and $1 \times 10^{-3}$ respectively, whereas 8-QAM and QAM provides BER in the order of $10^{-2}$. Comparing figure 10 and figure 11 , it can be said that the proposed OFDM scheme performs better in $3 \times 2$ MIMO configuration than in $2 \times 2$ configuration for all M-ary QAM modulation schemes.

The BER with respect to bit rate for $4 \times 3$ MIMO configuration at a constant SNR is plotted in Figure 12 . Here, QAM, 16-QAM and 32-QAM show poorest performance and 64-QAM shows the best performance (having BER of $2 \times 10^{-5}$ at $100 \mathrm{Mbps}$ bit rate).

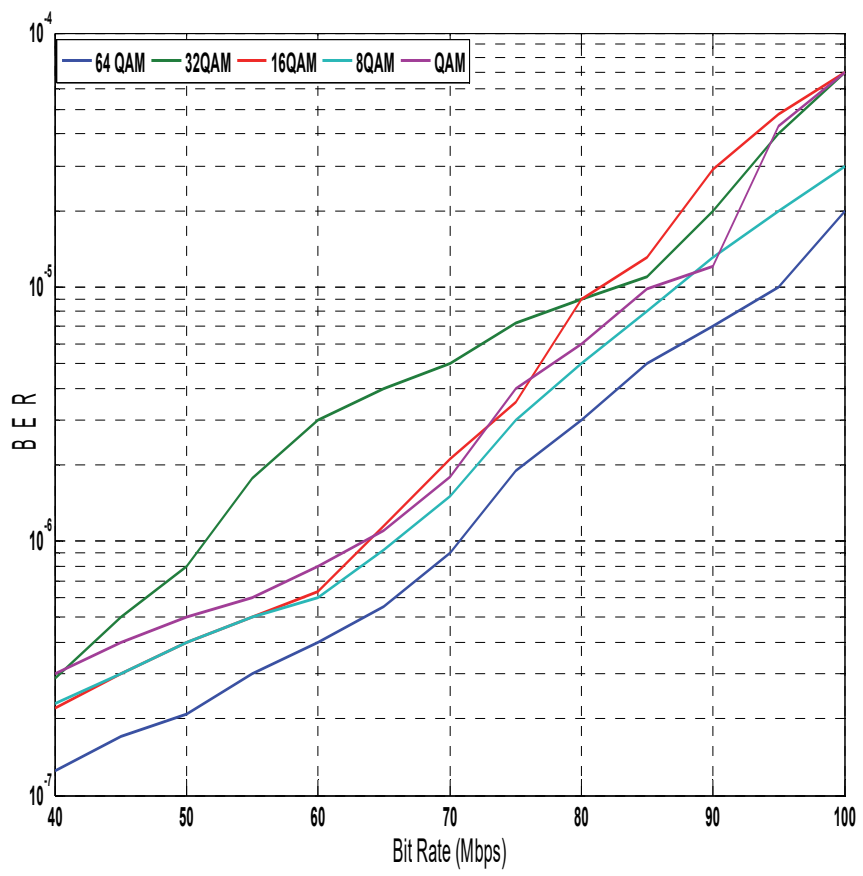

Figure 12. BER vs. Bit Rate curves of 4×3 MIMO-OFDM for different M-ary QAM modulation

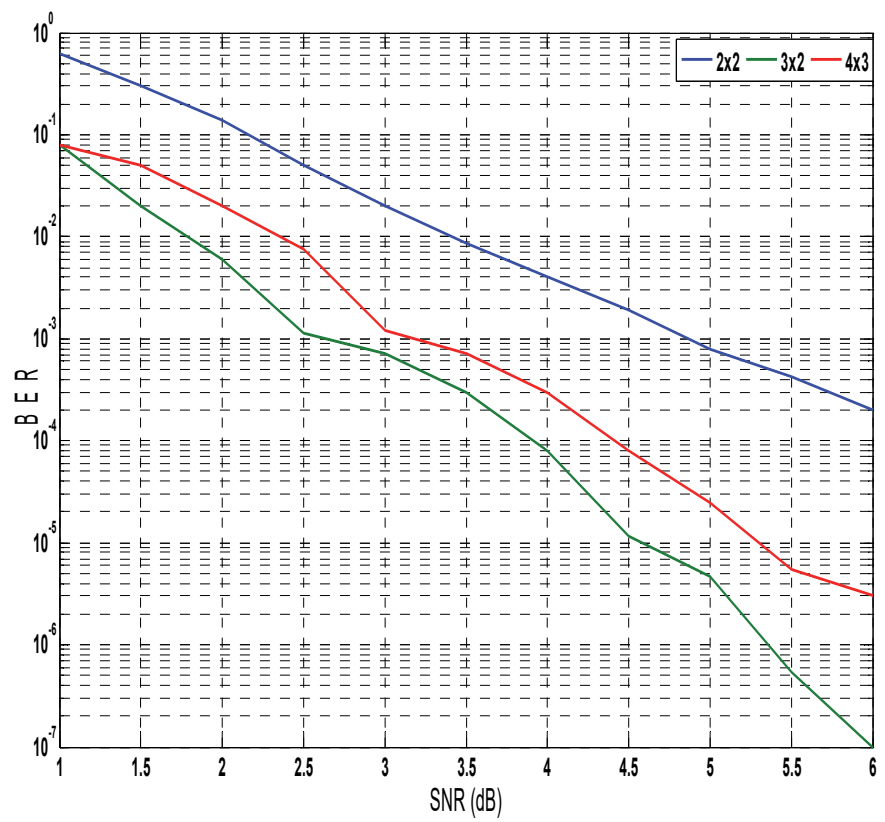

Figure 13. BER Vs SNR curves for different MIMO configurations of MIMO-OFDM using 64-QAM 
From the analysis, it is clearly realizable that the proposed MIMO-OFDM model performs better with 64-QAM than any other M-ary QAM modulation scheme. In order to find out which is the optimum MIMO configuration where 64-QAM modulation scheme performs better, the BER vs. SNR at a constant bit rate (figure 13) and BER vs. bit rate at a constant SNR (figure 14) are plotted using 64-QAM modulation only in all MIMO configurations investigated before. From these figures, it can be seen that $64-\mathrm{QAM}$ performs better in $3 \times 2 \mathrm{MIMO}$ configuration, than in $2 \times 2$ and $4 \times 3 \mathrm{MIMO}$.

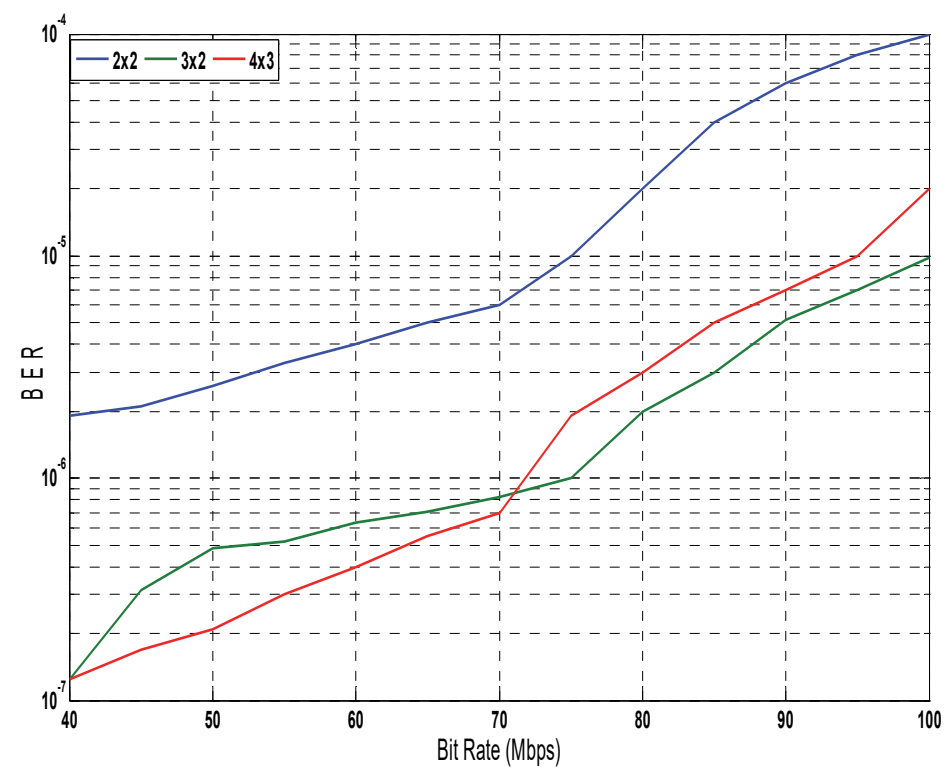

Figure 14. BER vs. Bit Rate curves for different MIMO configurations of MIMO-OFDM using 64-QAM

\section{Conclusion}

This paper presents a comprehensive analysis of a MIMO-OFDM model over Rayleigh Fading and AWGN channel using different M-ary QAM modulation schemes and different MIMO configurations in order to find out the best modulation scheme and the optimum MIMO configuration that provides minimum Bit Error Rate with high Bit Rate at low Signal to Noise Power Ratio for limited bandwidth spectrum. In order to maintain the frequency synchronization and the minimize Inter Carrier Interference of OFDM signal, the insertion of pilot, zero padding and cyclic prefix to the Fast Fourier Transform (FFT) operation is properly accomplished. After a thorough comparison of simulation results of the investigated MIMO configurations for different M-ary QAM modulation scheme, it is found that the MIMO-OFDM model with 64QAM for 3x2 antenna configuration provides the best BER performance which is approximately $10^{-7}$ for $6 \mathrm{~dB}$ SNR with a bit rate of $100 \mathrm{Mbps}$. This result will be useful for the RF researchers to have a comprehensive overview of the performance of the MIMO-OFDM technology and choose the optimum MIMO configuration and modulation scheme combination to have the required BER.

\section{Acknowledgments}

The authors would like to acknowledge the support of Chittagong University of Engineering and Technology towards this research.

\section{References}

Bhatnagar, P., Singh, J., \& Tiwari, M. (2011). Performance of MIMO-OFDM System for Rayleigh fading channel. International Journal of Science and Advanced Technology, 1(3), 28-32.

Dai, L., Wang, Z., \& Yang, Z. (2013). Spectrally efficient time-frequency training OFDM for mobile large-scale MIMO systems. IEEE Journal on Selected Areas in Communications, 31(2), 251-263. http://dx.doi.org/10.1109/JSAC.2013.130213

Dhore, K. S., \& Hate, S. G. (2015). Effect of multipath channel on OFDM symbol: guard interval approach. International Journal of Electronics and Communication Engineering \& Technology (IJECET), 6(6), 16-25. 
Gesbert, D., Shafi, M., Shiu, D. S., Smith, P. J., \& Naguib, A. (2003). From theory to practice: An overview of MIMO space-time coded wireless systems. IEEE Journal on selected areas in Communications, 21(3), 281-302. http://dx.doi.org/10.1109/JSAC.2003.809458

Gupta, B., Gupta, G., \& Saini, D. S. (2011). BER performance improvement in OFDM system with ZFE and MMSE equalizers. Proceedings of 3rd International Conference on Electronics Computer Technology (ICECT), Vol. 6, pp. 193-197. IEEE. http://dx.doi.org/10.1109/ICECTECH.2011.5942079

Jiang, M., \& Hanzo, L. (2007). Multiuser MIMO-OFDM for next-generation wireless systems. Proceedings of the IEEE, 95(7), 1430-1469. IEEE. http://dx.doi.org/10.1109/JPROC.2007.898869

Maharaja, N., Mishra, B. K., \& Bansode, R. (2016). Performance Evaluation of Spatial Multiplexing MIMO-OFDM System using MMSE Detection under Frequency Selective Rayleigh Channel. Global Journal of Computer Science and Technology: E Network, Web \& Security, 15(8).

Medvedev, I., Bjerke, B. A., Walton, R., Ketchum, J., Wallace, M., \& Howard, S. (2006). A comparison of MIMO receiver structures for $802.11 \mathrm{n}$ WLAN-performance and complexity. Proceedings of IEEE 17th International Symposium on Personal, Indoor and Mobile Radio Communications (pp. 1-5). IEEE. http://dx.doi.org/10.1109/PIMRC.2006.254135

Sahrab, A. A., \& Marghescu, I. (2014). MIMO-OFDM: Maximum diversity using maximum likelihood detector. Proceedings of 10th International Conference on Communications (COMM), (pp. 1-4). IEEE. http://dx.doi.org/10.1109/ICComm.2014.6866759

Sondhi, P., R. (2016). Performance Evaluation of Cyclic Prefix and Zero Padding Equalization for QPSK MIMO-OFDM System. International Journal of Modern Computer Science, 4(5), 72-75.

Tian, J., Jiang, H., Song, W., Luo, H., \& Xu, Y. (2006). Novel time-frequency differential space-time modulation for multi-antenna OFDM systems1. Journal of Systems Engineering and Electronics, 17(1), 54-58. https://doi.org/10.1016/S1004-4132(06)60010-8

Van De Beek, J. J., Edfors, O., Sandell, M., Wilson, S. K., \& Borjesson, P. O. (1995). On channel estimation in OFDM systems. Proceedings of IEEE 45th Vehicular Technology Conference, 1995 (Vol. 2, pp. 815-819). IEEE. http://dx.doi.org/10.1109/VETEC.1995.504981

Van Zelst, A., \& Schenk, T. C. (2004). Implementation of a MIMO OFDM-based wireless LAN system. IEEE Transactions on signal processing, 52(2), 483-494. http://dx.doi.org/10.1109/TSP.2003.820989

Wu, S., Kuang, L., Ni, Z., Lu, J., Huang, D., \& Guo, Q. (2014). Low-complexity iterative detection for large-scale multiuser MIMO-OFDM systems using approximate message passing. IEEE Journal of Selected Topics in Signal Processing, 8(5), 902-915. http://dx.doi.org/10.1109/JSTSP.2014.2313766

Yu, K., \& Ottersten, B. (2002). Models for MIMO propagation channels: a review. Wireless communications and mobile computing, 2(7), 653-666.

\section{Copyrights}

Copyright for this article is retained by the author(s), with first publication rights granted to the journal.

This is an open-access article distributed under the terms and conditions of the Creative Commons Attribution license (http://creativecommons.org/licenses/by/4.0/). 